\title{
Barriers and explanatory mechanisms in diagnostic delay in four cancers - A health-care disparity?
}

\author{
Abhishek Basu, Debjit Ghosh, Bidyut Mandal, Pratyusha Mukherjee',Avik Maji²
}

\begin{abstract}
Introduction: Most cancer disparities research has traditionally focused on two key outcomes, access to appropriate treatment and survival, but they do not encompass important aspects of patient-centered care such as the timeliness of diagnosis and treatment. Prolonged time intervals between symptom onset and treatment initiation increase the risk of poorer clinical outcomes and are associated with worse patient experience of subsequent cancer care. This study aims to assess the delay from symptom onset to the start of definitive treatment and to identify the possible contributory factors and its impact on response in cancers of head and neck, breast, cervix, and lung. Materials and Methods: This was a retrospective study of patients enrolled between 2015 and 2017. A questionnaire was filled in about socioeconomic aspects, patient history, tumor data, professionals who evaluated the patients, and the respective time delays. Statistical test included Mann-Whitney $U$ test, univariate and multivariate test, and one-way ANOVA to evaluate the correlations. Results: Stage migration was significant with patient delay $(P<0.0 \mathrm{I})$. In head and neck squamous cell carcinoma (HNSCC) and Carcinoma lung, a significant correlation was found between referral delay and residence $(P<0.0 \mathrm{I})$ and treatment delay and reason for referral $(\mathrm{HNSCC}$ only) $(P=0.04)$. Referral delay and treatment delay were correlated to response in breast and cervix, respectively $(P<0.0 \mathrm{I})$. Conclusion: Social awareness, regularly updating primary care physicians about alarming symptoms of cancer, developing guidelines to identify these symptoms, promoting continuity of care, and enabling access to specialist expertise through prompt referral should all help prevent delays in cancer diagnosis.
\end{abstract}

Key words: Barrier, diagnostic delay, four cancers, health care, impact

\section{Introduction}

Cancer is the leading cause of death in developed and developing countries. Cancer mortality is expected to rise to an estimated 13.1 million deaths annually by $2030 .{ }^{[1]}$ In India, over 7 lakhs new cancer patients are registered every year, and the estimated number of people living with the disease is around 2.5 million. According to the Indian Council of Medical Research report, India is likely to have over 17.3 lakh new cases of cancer and over 8.8 lakh deaths due to the disease by 2020 , with cancers of breast, lung, and cervix topping the list. According to the GLOBOAN 2018 cancers of breast, head neck (lip and oral cavity), cervix and lung predominates in the Indian Population. Cancers of oral cavity and lungs in males and cervix and breast in females account for over $50 \%$ of all cancer deaths in India. ${ }^{[2]}$ However, certain types of cancer have a high chance of cure if they are detected at an early stage and adequately treated. ${ }^{[3]}$ Most cancer disparities research has traditionally focused on two key outcomes, access to appropriate treatment and survival. While continuing research on these two measures remains critical, they do not encompass important aspects of patient-centered care such as the timeliness of diagnosis and treatment. Prolonged time intervals between symptom onset and treatment initiation increase the risk of poorer clinical outcomes and are associated with worse patient experience of subsequent cancer care. ${ }^{[4,5]}$

The delays in cancer diagnosis may occur throughout the diagnostic pathway: patient, primary care, and secondary care. Lesion location and the different forms of tumor presentation and symptoms may contribute to the delay. Silent tumors, those with difficult access, or those that take longer to manifest obvious symptoms hinder the patient's perception, delaying the entire diagnostic process. ${ }^{[6]}$ Therefore, very often, depending on the symptoms, it takes the patient longer to seek medical care.

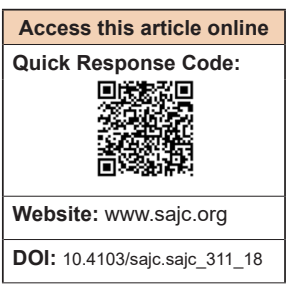

Department of Radiotherapy, Medical College, Kolkata, 'Department of Radiotherapy, IPGME and R, Kolkata, 2Diamond Harbour District Hospital, Diamond Harbour,West Bengal, India

Correspondence to: Dr.Abhishek Basu, E-mail: dr.abhishekbasul23@gmail.com
Studies have suggested increasing the awareness of individuals considered at risk, such as smokers and drinkers, to seek medical help after the initial symptoms, which favors prognosis. However, such delay may also be due to factors related to professional care and health care, with a time ranging in literature from 4 days to 3.5 months. ${ }^{[7]}$ The diagnostic workup is often a lengthy process, involving a complex algorithm and serial procedures (cytology, biopsy, immune histochemistry, and evaluation for distant metastases). If the time period between the initial consultation and treatment is prolonged, patients may experience tumor and clinical stage progression, which affects the therapeutic schedule with possible negative influence on prognosis. This is a relevant clinical problem, as comorbidity control before surgical treatment may require a long period. ${ }^{[8,9]}$ The literature regarding the length of diagnostic delays has several common themes. The effect of delays on clinical outcomes varies between cancers. In breast cancer, delays of 3-6 months are associated with poorer survival, although some patients who present early may have poorer outcomes. For some cancers, especially lung, morbidity and psychological outcomes may be more important than mortality. Psychological distress correlates positively with total diagnostic delay, itself a reason to minimize delays. ${ }^{[10]}$

This study analyses patients of four primary cancers: Head and neck, breast, cervix, and lung. This study aims to assess the delay from symptom onset to the start of definitive treatment and to identify the possible contributory factors and association between the delay and the tumor staging and response.

\section{Materials and Methods}

\section{Study design}

This is a retrospective study of new cases of squamous cell carcinoma of head and neck, carcinoma breast, carcinoma

This is an open access journal, and articles are distributed under the terms of the Creative Commons Attribution-NonCommercial-ShareAlike 4.0 License, which allows others to remix, tweak, and build upon the work non-commercially, as long as appropriate credit is given and the new creations are licensed under the identical terms.

For reprints contact: reprints@medknow.com

How to cite this article: Basu A, Ghosh D, Mandal B, Mukherjee P, Maji A. Barriers and explanatory mechanisms in diagnostic delay in four cancers - A health-care disparity? South Asian J Cancer 2019;8:221-5. 
cervix, and carcinoma lung, registered at the Outpatient Department (OPD) of Radiotherapy Department of Medical College Kolkata, from January 2015 to December 2017. Tumors of the salivary glands and thyroid were excluded from this study, as they have a different clinical presentation and biological behaviors from carcinoma of the head and neck region. Patients for whom primary definitive treatment was done outside and referred only for adjuvant or palliative treatment (chemotherapy or radiation) were excluded, but referrals within the home institution were included in the study.

\section{Data extraction}

Patients completed a questionnaire focused on epidemiological, disease, and treatment factors. Illiterate patients were aided by an accompanying family member and one of the authors was always available to resolve any doubts. The following data were collected: Identification (name, registration number at the institution, and telephone number), age (in years), gender, ethnicity, educational level (from none to College/University), smoking and alcohol consumption (both measured semiquantitatively), and primary tumor location. Tumors were staged according to the sixth edition of the tumor-node-metastasis classification of the American Joint Committee on Cancer.

\section{Recording and definition of variables}

The following durations were measured:

1. Interval between the reported symptom onset and seeking first medical care (patient delay, in months)

2. Interval between the first medical or dental appointment and the first consultation with the specialist (referral delay, in weeks)

3. Interval between first appointment with specialist and initiation of definitive treatment (treatment delay, in weeks).

Date of tissue diagnosis was defined as the date of final pathology report. Cause of death (cancer related versus other) was not available. Follow-up period was determined from the date of treatment completion to the last date of contact/death of the patient. Patients who did not complete the treatment and lost to follow-up were also excluded from the study. After the treatment, those who were terminally ill and unable to come to the OPD were contacted over telephone or via talking with the caregivers.

\section{Statistical analysis}

Statistical analysis was done using SPSS v23 software (IBM, SPSS makers, USA). Descriptive statistics (medians/ means for continuous variables and percentages for discrete variables) were generated for baseline demographic and clinical characteristics. Mann-Whitney $U$ test and univariate and multivariate Cox regression models were used to explore the association between time intervals and patient demographic and clinical characteristics. One-way ANOVA test was done to find the correlation between different delays and stage migration.

\section{Results}

In head and neck system, a total of 158 patients were analyzed. The median age was 53 years (range 26-71 years), predominantly male $(\mathrm{M}: \mathrm{F}=2.5: 1)$. Among the tumor sites, most frequent ones were oral cavity $(43.7 \%)>$ pharynx $(29.1 \%)$ $>\operatorname{larynx}(17.1 \%)>$ nasopharynx $(10.1 \%)$. The distribution of initial clinical stages shows a predominance of Stage II (median). The median time between onset of symptoms and seeking first medical care (patient delay) was 5 months, while the median referral delay and treatment delay were 2 weeks and 4 weeks, respectively [Table 1].

Patients had a median follow-up time of 16 months, and at the end of assessment, $44.3 \%$ of patients achieved complete response $(\mathrm{CR})$ while $17.1 \%$ of them reported either local or distant relapses. Among 158 patients, 27.2\% referred for definitive surgery, $16.5 \%$ for chemotherapy (neoadjuvant), $53.2 \%$ for radical radiotherapy, and $3.2 \%$ for palliative care [Table 2]. On univariate and multivariate analysis, significant correlations were found between patient delay and response $(P<0.001)$, patient delay and stage $(P=0.01)$, referral delay and residence $(P=0.004)$, treatment delay and reason for referral $(P=0.04)$, and treatment delay and tumor site $(P<0.001)$.

In breast cancer system, 252 women were followed up for a median time of 14 months. The median age of the patients was 44 years and $65.4 \%$ of the cases belong to adult and young adult group [Table 3]. Most common stage observed was Stage III. The median time for patient delay, referral delay, and treatment delay were 4 months, 3 weeks, and 4 weeks, respectively. It was observed that $75 \%$ of patients had CR while $19.7 \%$ of the patients had local or distant failures at the end of follow-up [Table 1]. Stage migration was related to patient delay $(P=0.002)$ and referral delay $(P=0.03)$. A significant correlation was observed between tumor response and referral delay $(P=0.004)$.

In CA cervix subgroup, 286 women were followed up for a median time of 20 months [Table 3]. Most common stage observed was Stage IIIB. The median time for patient delay, referral delay, and treatment delay were 3 months, 3 weeks, and 4 weeks, respectively. Most of the patients were referred for radical radiotherapy (concomitant chemoradiation) (92\%), while few of them were referred for upfront surgery, chemotherapy, or palliative care [Table 2]. On analysis, it showed that $62.9 \%$ of women completed treatment within 56 days from the start of radiotherapy, while $37.1 \%$ women achieved that beyond 56 days. About $80.1 \%$ of patients achieved CR while $7.7 \%$ had local or distant failures at the end of follow-up [Table 1]. On multivariate analysis and one-way ANOVA, a significant correlation was found between response and stage $(P<0.001)$, response and treatment delay $(P=0.006)$, and response and overall treatment time $(P<0.001)$. Stage migration with patient delay was found to be statistically significant.

Our study included 149 patients of lung carcinoma, of which 102 were nonsmall cell lung cancer (NSCLC) and 47 cases were small cell lung cancer (SCLC). It was surprising to observe that $19.8 \%$ of patients had taken alternative medications before consulting a physician. The median time for patient delay, referral delay, and treatment delay were 4 months, 5 weeks, and 4 weeks, respectively. Most of the patients were referred for chemotherapy $>$ palliative care $>$ chemoradiation $>$ surgery [Table 2]. After a median period of 11 months, it was found that most common stage for NSCLC was Stage III (41.6\%), and for SCLC, it was limited stage $(16.8 \%)$. 
Table 1: Median and range of the parameters studied

\begin{tabular}{|c|c|c|c|c|c|c|c|}
\hline \multirow[t]{2}{*}{ System } & \multirow[t]{2}{*}{ Stage } & \multicolumn{5}{|c|}{ Median values (range) } & \multirow{2}{*}{$\begin{array}{l}\text { Recurrence } \\
\text { (local and } \\
\text { distant) }(\%)\end{array}$} \\
\hline & & $\begin{array}{c}\text { Patient } \\
\text { delay (month) }\end{array}$ & $\begin{array}{c}\text { Referral } \\
\text { delay (weeks) }\end{array}$ & $\begin{array}{c}\text { Treatment } \\
\text { delay (week) }\end{array}$ & $\begin{array}{c}\text { PS } \\
(\mathrm{ECOG})\end{array}$ & $\begin{array}{c}\text { FU } \\
\text { months }\end{array}$ & \\
\hline Head and neck & II & $5(3-7)$ & $2(2-5)$ & $4(2-6)$ & $1(0-2)$ & $16(8-24)$ & 17.1 \\
\hline Breast & III & $4(2-6)$ & $3(2-6)$ & $4(2-8)$ & $1(0-3)$ & $14(7-26)$ & 19.7 \\
\hline Cervix & IIIB & $3(1-5)$ & $3(2-8)$ & $4(3-7)$ & $1(0-3)$ & $20(9-24)$ & 7.7 \\
\hline Lung & III & $4(2-8)$ & $5(3-9)$ & $4(2-9)$ & $3(1-4)$ & $11(7-18)$ & 22.8 \\
\hline
\end{tabular}

ECOG=Eastern Cooperative Oncology Group, PS=Performance status, FU=Follow-up

Table 2: Reason for referral (percentage)

\begin{tabular}{lcccc}
\hline System & Surgery & Chemotherapy & Radical radiotherapy & Palliative care \\
\hline Head and neck & 27.2 & 16.5 & 53.2 & 3.2 \\
Breast & 33.3 & 47.6 & 0 & 17.8 \\
Cervix & 2.8 & 2.1 & 92 & 3.1 \\
Lung & 4 & 66.4 & 6.7 & 22.8 \\
\hline
\end{tabular}

Table 3: Epidemiological data of the assessed patients

\begin{tabular}{lccccc}
\hline System & Age (years), median & AYA (\%) & Rural/urban (\%) & Addiction (\%) & Alternative medicine (\%) \\
\hline Head and neck (158) & 54 & 39 & $57 / 43$ & 69.5 & 17.1 \\
Breast (252) & 44 & 65.4 & $63.4 / 36.6$ & 8.3 & 3.6 \\
Cervix (286) & 53 & 44.7 & $65.4 / 34.6$ & 23 & 5.2 \\
Lung (149) & 59 & 22.1 & $67.1 / 32.9$ & 80.5 & 19.8 \\
\hline
\end{tabular}

AYA=Adult-young adult

A significant correlation was found between tumor response and referral delay $(P<0.001)$ and treatment delay $(P=0.02)$ and stage $(P<0.01)$. Referral delay was related with residence $(P<0.01)$ and performance status $(P<0.001)$. One-way ANOVA showed a significant correlation between stage and patient delay $(P=0.03)$. The overall tumor response was related to total delay $(P<0.01)$, but not with patient delay alone. Alternative medications and other demographic factors did not influence the treatment outcome significantly.

\section{Discussion}

\section{Statement of principal findings}

This study reports findings from the analysis of a large data set of patients relating to the barriers and explanatory mechanisms of delays and their impact on tumor response and stage migration. Total diagnostic delays remain long, particularly in some cancers. Our study found that the mean total delay was greatest for CA lung and was minimum for CA cervix, followed by $\mathrm{CA}$ breast and head and neck squamous cell carcinoma (HNSCC) [Figure 1]. Tumor response was better in cervix and breast subgroup where it was worse in head and neck and CA lung patients [Figure 2]. The median patient delay was higher in head and neck and lung cancer patients than CA cervix and CA breast. This delay can be, in part, attributed to specific patient factors. The population's difficulty in accessing a specialist seems to be due in part to their low socioeconomic status. Sometimes, they lack funds even for the use of public transport. Patient delay, as mentioned before, is difficult to measure accurately because it is based on perception, which is highly subjective and can be influenced by many social and cultural factors. ${ }^{[1]}$ In addition, when considering the entire case management, patients should also be carefully instructed about the return visits and follow-up. Longer patient delay may be attributed to the different sites of HNSCC and symptoms of low predictive value in CA lung. For example, possible lung cancer symptoms such as persistent cough and dyspnea have low specificity and are difficult to distinguish South Asian Journal of Cancer • Volume 8 • Issue 4 October-December 2019

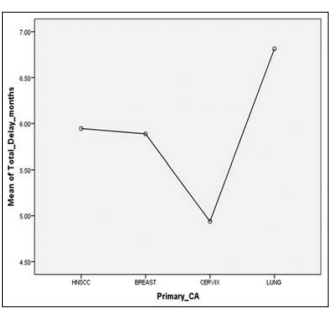

Figure 1: Means of total delay and types of primary cancer

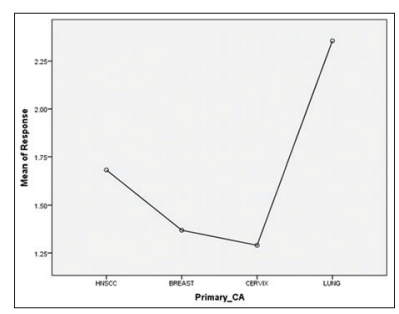

Figure 2: Means of response and types of primary cancer from manifestations of chronic pulmonary disease in smokers. Conversely, hemoptysis, a classic red flag' symptom with relatively high predictive value, only occurs in a minority of patients. ${ }^{[12]}$

Referral delay was less in HNSCC while it was greater in CA breast and CA lung. This delay could be attributed to residence, performance status, time required for confirmation of diagnosis, and metastatic workup. Reasons for referral could also play some part while this study did not find any such significant correlations. Another important factor was taking alternative medications such as homeopathy or Ayurveda, patients of CA lung (19.8\%) and CA head and neck (17.1\%) were highest in the study population to take alternative medications. Although it did not reflect any statistical significance in terms of stage migration or response, relatively small sample size could be a reason for that. Treatment delay was more or less uniform in all subgroups though the range of values was greater in CA lung group owing to poor performance status and availability of beds (for chemotherapy) in a high output center like us.

Meaning of the study: Possible explanations and implications for clinicians and policymakers

In a systematic review in head and neck malignancy, Withers et al. ${ }^{[13]}$ provided the first clinical evidence that if the overall treatment time was prolonged, the observed Loco-regional control (LRC) loss would have required an average increase of $0.6 \mathrm{~Gy} /$ day to compensate for it. In addition, the review by 
Fowler and Lindstrom ${ }^{[14]}$ revealed a median value of $14 \%$ of LRC loss per week of extra overall time. ${ }^{[15]}$

Despite formal recommendations, the impact of diagnostic and treatment delays on the outcomes of patients with NSCLC remains unclear. ${ }^{[16]}$ A number of professional organizations have put forth specific recommendations regarding the optimal timing of lung cancer diagnosis and treatment. The Swedish Lung Cancer Study Group recommends that treatment should be initiated within 42 days of suspicious imaging. ${ }^{[17]}$ The British Thoracic Society recommends that no more than 70 days elapse between radiograph and thoracotomy. ${ }^{[18,19]}$ Radzikowska et al. showed that the delay in the diagnosis and treatment had no effect on survival in SCLC patients. Interestingly, patients who were diagnosed faster (below 42 days) actually had a worse prognosis than those diagnosed later. The median delay was 30 days (mean 47 days), and the median referral delay to a specialist was 19 days (mean 36 days). Half of SCLC patients were diagnosed during 34 days (mean 55 days). The mean time elapse from the diagnosis to the onset of therapy was 30 days (median 6 days). The multivariate analysis revealed that male gender (hazard ratio $[\mathrm{HR}]=1.2$ ), Eastern Cooperative Oncology Group performance status of $2(\mathrm{HR}=1.5)$ and $3+4(\mathrm{HR}=2.4)$, and clinical Stage III $(\mathrm{HR}=1.3)$ and IV $(\mathrm{HR}=1.9)$ of the disease were independent negative predictors of survival..$^{[16,20,21]}$

Shorter delays in breast and cervix cancer compared with other cancers may occur because of a more straightforward presentation of signs and symptoms that are easily understood by patients and doctors, clear referral guidance, well-organized secondary care clinics, a national screening program, and a high public profile. Reductions in delays may improve survival. Delays may improve in the future with more opportunistic screening, although the effect of this on survival is unknown. While there is insufficient evidence at present to prove that shorter delays are associated with better prognosis, there is considerable logic that this should be the case, given the potential for curative treatments. ${ }^{[10,22-24]}$

The finding that patient delays were the longest suggests that while further reductions in referral delays and treatment delays may result in better psychological outcomes, attempts to improve clinical outcomes (earlier stage diagnosis and improved survival), must be directed at patient and/or primary care delays. ${ }^{[2]}$

The diagnostic delay has a significant impact on the intent of treatment eventually received by patients. Delays on behalf of the patient and primary care physician have a significant contribution to overall delay. Apart from educating the population regarding cancer symptoms, increasing cancer awareness among primary care physicians and initiating chemotherapy at district level might improve overall diagnostic delay. ${ }^{[26]}$

\section{Limitations of the study}

It was a retrospective, single institutional study. There is the potential for recall bias, especially given the time interval between diagnosis and survey completion, for at least some of the sample. Data were not collected relating to comorbidity, histological type of cancer, or the natural history of cancer; hence, we cannot be sure that the sample was representative of the "cancer population," and cannot exclude the possibility of confounding as a result.
Unanswered questions and future research

Before the development and evaluation of interventions to reduce delay, ${ }^{[16]}$ further work needs to be performed to elucidate the separate contributions of patient and primary care delays to the overall delays. There may be variation between delays and sociodemographic factors, and local or regional variations; these need quantifying before intervention. Findings from the ever-increasing evidence base on the reasons for patient delays in most cancers, and the smaller evidence base regarding primary care delays ${ }^{[10,27]}$ will inform the development of the interventions.

\section{Conclusion}

A delay in cancer diagnosis can occur at various levels. The patient may fail to recognize suspicious cancer symptoms or act on them. The primary care physician may not recognize patients with suspicious cancer symptoms and investigate them appropriately or refer on time. Patients with suspicious cancer in secondary care may not be seen on time, or they may be referred to the wrong specialty. Thus, using media to broadcast the message of awareness in the community should increase public knowledge of cancer symptoms and the importance of seeking timely medical attention. Regularly updating primary care physicians about alarming symptoms of cancer, developing guidelines to identify these symptoms, promoting continuity of care, and enabling access to specialist expertise through prompt referrals should all help to prevent delays in cancer diagnosis.

\section{Acknowledgment}

Colleagues of Dept of Radiotherapy, MCH, KOLKATA and patients of this study for their utmost cooperation.

\section{Financial support and sponsorship}

Nil.

\section{Conflicts of interest}

There are no conflicts of interest.

\section{References}

1. World Health Organization. Cancer- Fact Sheet. World Health Organization; 2014. Available from: http://www.who.int/mediacentre/ factsheets/fs297/en. [Last accessed on 2016 May 09].

2. National Institute of Cancer Prevention and Research. Cancer Statistics Fact Sheet. National Institute of Cancer Prevention and Research; 2018. Available from: http://www.cancerindia.org.in/statistics/. [Last accessed on 2018 Jun 06].

3. Harford JB. Breast-cancer early detection in low-income and middle-income countries: Do what you can versus one size fits all. Lancet Oncol 2011;12:306-12.

4. Neal RD, Tharmanathan P, France B, Din NU, Cotton S, Fallon-Ferguson J, et al. Is increased time to diagnosis and treatment in symptomatic cancer associated with poorer outcomes? Systematic review. Br J Cancer 2015;112 Suppl 1:S92-107.

5. Koo MM, Zhou Y, Lyratzopoulos G. Delays in diagnosis and treatment of lung cancer: Lessons from US healthcare settings. Cancer Epidemiol 2015;39:1145-7.

6. Brouha XD, Tromp DM, Koole R, Hordijk GJ, Winnubst JA, de Leeuw JR, et al. Professional delay in head and neck cancer patients: Analysis of the diagnostic pathway. Oral Oncol 2007;43:551-6.

7. Goy J, Hall SF, Feldman-Stewart D, Groome PA. Diagnostic delay and disease stage in head and neck cancer: A systematic review. Laryngoscope 2009;119:889-98.

8. Tromp DM, Brouha XD, De Leeuw JR, Hordijk GJ, Winnubst JA. Psychological factors and patient delay in patients with head and neck cancer. Eur J Cancer 2004;40: 1509-16.

9. Diaconescu R, Lafond C, Whittom R. Treatment delays in non-small cell lung cancer and their prognostic implications. J Thorac Oncol 2011;6:1254-9.

10. Allgar VL, Neal RD. Delays in the diagnosis of six cancers: Analysis of data from the national survey of NHS patients: Cancer. Br J Cancer 
2005;92: 1959-70

11. Carvalho AL, Pintos J, Schlecht NF, Oliveira BV, Fava AS, Curado MP, et al. Predictive factors for diagnosis of advanced-stage squamous cell carcinoma of the head and neck. Arch Otolaryngol Head Neck Surg 2002; 128:313-8.

12. Walter FM, Rubin G, Bankhead C, Morris HC, Hall N, Mills K, et al. Symptoms and other factors associated with time to diagnosis and stage of lung cancer: A prospective cohort study. Br J Cancer 2015;112 Suppl 1:S6-13.

13. Withers HR, Taylor JM, Maciejewski B. The hazard of accelerated tumor clonogen repopulation during radiotherapy. Acta Oncol 1988;27:131-46.

14. Fowler JF, Lindstrom MJ. Loss of local control with prolongation in radiotherapy. Int J Radiat Oncol Biol Phys 1992;23:457-67.

15. González Ferreira JA, Jaén Olasolo J, Azinovic I, Jeremic B. Effect of radiotherapy delay in overall treatment time on local control and survival in head and neck cancer: Review of the literature. Rep Pract Oncol Radiother 2015;20:328-39.

16. Jensen AR, Mainz J, Overgaard J. Impact of delay on diagnosis and treatment of primary lung cancer. Acta Oncol 2002;41:147-52.

17. Myrdal G, Lambe M, Hillerdal G, Lamberg K, Agustsson T, Ståhle E, et al. Effect of delays on prognosis in patients with non-small cell lung cancer. Thorax 2004;59:45-9.

18. BTS recommendations to respiratory physicians for organising the care of patients with lung cancer. The Lung Cancer Working Party of the British Thoracic Society Standards of Care Committee. Thorax 1998;53 Suppl 1:S1-8.

19. Yorio JT, Xie Y, Yan J, Gerber DE. Lung cancer diagnostic and treatment intervals in the United States: A health care disparity? J Thorac Oncol 2009; 4: 1322-30.

20. Radzikowska E, Roszkowski-Sliz K, Chabowski M, Glaz P. Influence of delays in diagnosis and treatment on survival in small cell lung cancer patients. Adv Exp Med Biol 2013;788:355-62.

21. Basu A, Mandal B, Ghosh D, Maji A. Patterns of care and factors affecting it in small cell lung cancer - Analysis of 3 years data from a tertiary care hospital in India. J Cancer Tumor Int 2018;7:1-7.

22. Richards MA, Smith P, Ramirez AJ, Fentiman IS, Rubens RD. The influence on survival of delay in the presentation and treatment of symptomatic breast cancer. Br J Cancer 1999;79:858-64.

23. Richards MA, Westcombe AM, Love SB, Littlejohns P, Ramirez AJ. Influence of delay on survival in patients with breast cancer: A systematic review. Lancet 1999;353:1119-26.

24. Nosarti C, Crayford T, Roberts JV, Elias E, McKenzie K, David AS, et al. Delay in presentation of symptomatic referrals to a breast clinic: Patient and system factors. Br J Cancer 2000;82:742-8.

25. Risberg T, Sørbye SW, Norum J, Wist EA. Diagnostic delay causes more psychological distress in female than in male cancer patients. Anticancer Res 1996; 16:995-9.

26. Miriyala R, Bansal A, Dracham C, Thakur P, Ghoshal S. Diagnostic delay in oncology: Is there a need for increasing cancer awareness among primary care physicians of developing countries? Ann Oncol 2016;27 Suppl 9:ix 184-9.

27. Spellman P, Smith I, Bruce E, Neal RD. The Cancer Journey to Secondary Care of Patients with Suspected Cancer of the Colon or Lung: A Perspective from General Practice. Leeds: Nuffield Portfolio Programme; 1999. 